\title{
Erratum to: PEGylated PAMAM Dendrimer-Doxorubicin Conjugates: In Vitro Evaluation and In Vivo Tumor Accumulation
}

Saijie Zhu • Minghuang Hong • Lihong Zhang • Guotao Tang • Yanyan Jiang $•$ Yuanying Pei

Published online: 20 July 2010

(C) Springer Science+Business Media, LLC 2010

Erratum to: Pharm Res

DOI | 0.1007/s | 1095-009-9992-1

\section{NOTICE OF DUPLICATE PUBLICATION}

Our recent article published in Pharmaceutical Research (1) was prepared simultaneously with an article published in Biomaterials (2), both examining the effects of an identical batch of PEGylated PAMAM dendrimer-doxorubicin conjugates on tumor accumulation. The polymer synthesis and characterization sections in both articles were inadvertently duplicated, namely Figures 1 and 2 in Pharmaceutical Research and Figures 2 and 3 in Biomaterials.

We would like to apologize to the editors and readers of Biomaterials and Pharmaceutical Research for any ambiguity this may have caused.

\section{REFERENCES}

Zhu S, Hong M, Zhang L, Tang G, Jiang Y, Pei Y. PEGylated PAMAM dendrimer-doxorubicin conjugates: in vitro evaluation and in vivo tumor accumulation. Pharm Res. 2010a;27(1):161-74. Submitted: July 27, 2009.

Zhu S, Hong M, Tang G, Qian L, Lin J, Jiang Y, et al. Partly PEGylated polyamidoamine dendrimer for tumor-selective targeting of doxorubicin: the effects of PEGylation degree and drug conjugation style. Biomaterials. 2010b;31(6):136071. Submitted: August 21, 2009.
The online version of the original article can be found at http://dx.doi.org/ | 0.1007/s | |095-009-9992-I.

S. Zhu $\cdot$ M. Hong $\cdot$ L. Zhang $\cdot$ G. Tang $\cdot$ Y. Jiang $(\bowtie) \cdot$ Y. Pei

Department of Pharmaceutics, School of Pharmacy, Fudan University

826 Zhangheng Road

Shanghai 201203, China

e-mail: yanyanjiang@shmu.edu.cn 